\title{
Physico-chemical State of the Air at the Stagnation Point during the Atmospheric Reentry of a Spacecraft
}

\author{
Rabah Haoui
}

\begin{abstract}
Hypersonic flows around spatial vehicles during their reentry phase in planetary atmospheres are characterized by intense aerothermal phenomena. The aim of this work is to analyze high temperature flows around an axisymmetric blunt body taking into account chemical and vibrational non-equilibrium for air mixture species. For this purpose, a finite volume methodology is employed to determine the supersonic flow parameters around the axisymmetric blunt body, especially at the stagnation point and along the wall of spacecraft for several altitudes. This allows the capture shock wave before a blunt body placed in supersonic free stream. The numerical technique uses the Flux Vector Splitting method of Van Leer. Here, adequate time stepping parameter, along with CFL coefficient and mesh size level are selected to ensure numerical convergence, sought with an order of $\mathbf{1 0}^{\mathbf{- 8}}$
\end{abstract}

Keywords-Chemical kinetic, dissociation, finite volumes, frozen, hypersonic flow, non-equilibrium, Reactive flow, supersonic flow, vibration.

\section{INTRODUCTION}

$\mathrm{T}$ HIS article presents a calculation of a reactive flow around an axisymmetric blunt body, hemisphere-cylinder. Experiments for the hypersonic shock tunnel remain a valuable source of information on the physicochemical phenomena which occur in the flow of air surrounding the model. Numerical simulations came to supplement the theoretical and experimental studies thanks to the appearance of powerful calculators and with the development of powerful mathematical tools. In the present work, we implemented a numerical technique to simulate the hypersonic flow around an axisymmetric blunt body. The gas considered is the air in a standard state composed of $21 \%$ of $\mathrm{O}_{2}$ and $79 \%$ of $\mathrm{N}_{2}$. In this case, the infinite conditions of the flow are those of the atmosphere at a given altitude. In addition, it is necessary to take into account the air non-equilibrium state at the exit of a nozzle to compare with experiment data Haoui [3].

Upstream the body, an intense detached shock wave occurs resulting in a very large temperature raise located at the nose of the body just after the shock. In this case the air starts to dissociated and transforms gas composed of five species, $\mathrm{O}_{2}$, $\mathrm{N}_{2}, \mathrm{NO}, \mathrm{O}$ and $\mathrm{N}$. There are several models treating the chemical kinetics that differ according to the number of chemical reactions taken into account.

Haoui Rabah is with University of Science and Technology Houari Boumediene, Algiers. e-mail:tec374@yahoo.fr
A realistic study must consider all the probable chemical reactions occurring in the flow. Here, the model with seventeen chemical reactions is selected, allowing for all possible reactions between chemical species constituting the mixture [3].

Speeds of the reactions employed are those of Arrhenius law. This is in addition to the expression of Landau Teller [5] for non-equilibrium of vibration. Time characteristic of vibration is given by Blackman model [6]. Time characteristic of translation and rotation of molecules are very short comparing to time characteristic transition of the flow, whilst, the return to equilibrium state for these modes is very fast. Note that equilibrium of translation and rotation is carried out in any point of the flow and at any moment. By considering the range of temperatures in which the present study is placed, the phenomena of ionization of the species can be neglected. Similarly, radiation effect is ignored. The chemical kinetics and the non-equilibrium of vibration are taken into account in the computational domain. The system of nonlinear partial derivative equations which governs this flow are solved by an explicit unsteady numerical scheme.

\section{EQUATIONS}

The Euler equations for the mixture in non-equilibrium contain in addition for mass conservation, momentum and energy, equations of the evolution of chemical species $\left(\mathrm{N}_{2}, \mathrm{O}_{2}\right.$, $\mathrm{NO}, \mathrm{O}, \mathrm{N})$ and the energy of vibration of the molecules. It should be noted that only $\mathrm{O}_{2}$ and $\mathrm{N}_{2}$ species are considered in non-equilibrium state. The molecule of $\mathrm{NO}$, is taken in equilibrium of vibration as its time characteristic of vibration is of two orders of magnitude lower than that of $\mathrm{O}_{2}$ between $3000 \mathrm{~K}$ and $7000 \mathrm{~K}[7]$.

In its vectorial form, the system of equations is written in $3 \mathrm{D}$ as following:

$$
\frac{\partial W}{\partial t}+\operatorname{div}(f)=\Omega
$$

Where:

$$
\vec{f}=F \cdot \vec{i}+G \cdot \vec{j}+H \cdot \vec{k}
$$

The flux $W, F, G, H$ and $\Omega$ are: 
$W=\left\{\begin{array}{l}\rho \\ \rho u \\ \rho v \\ \rho w \\ \rho e \\ \rho_{s} \\ \left(\rho_{s} e_{v s}\right)_{O 2, N 2}\end{array}\right\} \quad F=\left\{\begin{array}{l}\rho u \\ \rho u^{2}+p \\ \rho u v \\ \rho u w \\ (\rho e+p) u \\ \rho_{s} u \\ \left(\rho_{s} e_{v s} u\right)_{O 2, N 2}\end{array}\right\}$

$$
G=\left\{\begin{array}{l}
\rho v \\
\rho u v \\
\rho v^{2}+p \\
\rho v w \\
(\rho e+p) v \\
\rho_{s} v \\
\left(\rho_{S} e_{v s} v\right)_{O 2, N 2}
\end{array}\right\} \quad H=\left\{\begin{array}{l}
\rho w \\
\rho u w \\
\rho v w \\
\rho w^{2}+p \\
(\rho e+p) w \\
\rho_{S} w \\
\left(\rho_{S} e_{v s} w\right)_{O 2, N 2}
\end{array}\right\}
$$

$$
\Omega=\left\{\begin{array}{l}
0 \\
0 \\
0 \\
0 \\
0 \\
\omega_{C S} \\
\left(\omega_{v S}\right)_{O 2, N 2}
\end{array}\right\}
$$

The energy per unit of mass $\boldsymbol{e}$ is such as:

$$
e=\sum_{s=1}^{5} c_{v s} T+\sum_{s=1}^{5} Y_{s} e_{v s}+\sum_{s=1}^{5} Y_{s} h_{f}^{0}+\frac{1}{2}\left(u^{2}+v^{2}+w^{2}\right)
$$

$h_{f}^{0}$ is the enthalpy of formation of the species $s$ in $\mathrm{J} / \mathrm{kg}$ :

$$
\begin{gathered}
h_{f}^{0}(O)=15.4310^{6}, \quad h_{f}^{0}(N)=33.6210^{6} \\
h_{f}^{0}(N O)=2.99610^{6} \\
h_{f}^{0}(O 2)=h_{f}^{0}(N 2)=0
\end{gathered}
$$

The pressure of the mixture is obtained by the equation of state:

$$
\frac{p}{\rho}=r_{m} T
$$

Where

$$
r_{m}=\frac{R}{M_{m}}
$$

The temperature of the mixture is calculated based on the energy equation (5). The source term of the chemical equation of evolution of the species $s$ is given provided through:

$$
\omega_{c s}=M_{s} \sum_{r=1}^{r}\left(v_{s}^{\prime \prime}-v_{s}^{\prime}\right) J_{r}
$$

Where:

$$
\begin{aligned}
& J_{r}=K_{f} \prod_{s}\left(\frac{\rho_{s}}{M_{s}}\right)^{v_{s}^{\prime}}-K_{b} \prod_{s}\left(\frac{\rho_{s}}{M_{s}}\right)^{v_{s}^{\prime \prime}} \\
& v_{s}^{\prime} \text { and } v_{s}^{\prime} \text { are the stoichiometric mole numbers of the }
\end{aligned}
$$
reactants and products of species $s$, respectively, for each chemical reaction $(r)$ such as:

$$
\left.\sum_{s} v_{s}^{\prime} A_{s} \stackrel{K_{b}^{K}}{\Leftrightarrow} \sum_{s} v_{s}^{\prime \prime} A_{s} \quad \text { (reactin } r\right)
$$

Both forward and backward reaction rates are represented by $K_{f}$ and $K_{b}$. An empirically expression for the forward reaction rate $K_{f}$ may be written as

$$
K_{f}=A T^{n} \exp \left(-\frac{T_{d}}{T}\right)
$$

For the backward reaction rate $K_{b}$, it is function of the equilibrium constant $K_{e q}$

$$
K_{b}=\frac{K_{f}}{K_{e q}}
$$

The constants $A, n$ and the temperature characteristic of dissociation $T_{d}$ are given by Gardiner model [8], Table I. The equilibrium constant of the chemical reaction is given like a polynomial of the $4^{\text {th }}$ degree:

$$
K_{e q}=\exp \left(c_{0}+c_{1} z+c_{2} z^{2}+c_{3} z^{3}+c_{4} z^{4}\right)
$$

Where $z=10000 / T$, and the coefficients $c_{0}$ through $c_{4}$ are provided for each reaction [8], see Table II. 
The term of energy production of vibration $\omega_{v s}$ is such as:

$$
\omega_{v s}=\rho_{S} \frac{e_{v}(T)-e_{v}\left(T_{v}\right)}{\tau_{s}}+e_{v S} \omega_{c S}
$$

$e_{V}(T) \quad$ is the equilibrium energy of vibration at the temperature of translation-rotation expressed as:

$$
e_{v}(T)=\frac{r \cdot \theta_{v}}{\exp \left(\frac{\theta_{v}}{T}\right)-1}
$$

$r$ is the constant of a particular gas and $\theta_{v}$ is the temperature characteristic of vibration. It is specified for each molecule. In the present work:

$$
\theta_{v O 2}=2239 K \quad \theta_{v N 2}=3354 K \quad \theta_{v N O}=2720 K
$$

$e_{v}\left(T_{v}\right)$ is the energy at the temperature of vibration $T_{v}$. The time characteristic of vibration of a species $s$ in the mixture $\tau_{s}$ is function of the temperature, the pressure and the molar fractions $X_{i}$. It can be calculated as follows [9]:

$$
\frac{1}{\tau_{s}}=\sum_{i=1}^{5} \frac{X_{i}}{\tau_{s, i}}
$$

Where $s=O_{2}, N_{2}$ and $i=O_{2}, N_{2}, N O, O, N . \tau_{s, i}$ is the time characteristic of vibration of the species $s$ in a mixture containing species $i$. We have:

$$
\begin{gathered}
\tau_{O 2, O 2}=\frac{1.69210^{-9}}{p} \exp \left(101.44 T^{-1 / 3}\right) \\
\tau_{N 2, N 2}=\frac{1.110^{-11}}{p} T^{1 / 2} \exp \left(154.0 T^{-1 / 3}\right)
\end{gathered}
$$

Experiments for binary exchanges, made it possible to evaluate vibrational relaxation time of $\mathrm{O}_{2}$ in monatomic oxygen - see work of Kiefer and Lutz [10] - and of $N_{2}$ in $O$ see work of Brashears and Bird [11] -, both correlated by Thivet [12]. The pressure $p$ is in atmospheres and $\tau$ in seconds.

$$
\begin{aligned}
\tau_{O 2, O} & =\frac{T^{2 / 3}}{p} \exp \left(55.2 T^{-1 / 3}-26.95\right) \\
\tau_{N 2, O} & =\frac{T^{2 / 3}}{p} \exp \left(70.3 T^{-1 / 3}-24.35\right)
\end{aligned}
$$

To determine the relaxation times which are not given by the experimental correlations, is supposed that the relaxation time of a species $s$ is same whatever the species $i$ which it meets, provided that their masses are identical. Therefore, the following approximations can be employed:

$$
\begin{gathered}
\tau_{O 2, N} \cong \tau_{O 2, O} \quad \tau_{N 2, N} \cong \tau_{N 2, O} \\
\tau_{O 2, N 2} \cong \tau_{O 2, N O} \cong \tau_{O 2, O 2} \\
\tau_{N 2, O 2} \cong \tau_{N 2, N O} \cong \tau_{N 2, N 2}
\end{gathered}
$$

The model with 17 reactions is:

$$
\begin{array}{ll}
\mathrm{O}_{2}+\mathrm{M} \leftrightarrow 2 \mathrm{O}+\mathrm{M} & \mathrm{r}=1 \text { to } 5 \\
\mathrm{~N}_{2}+\mathrm{M} \leftrightarrow 2 \mathrm{~N}+\mathrm{M} & \mathrm{r}=6 \text { to } 10 \\
\mathrm{NO}+\mathrm{M} \leftrightarrow \mathrm{N}+\mathrm{O}+\mathrm{M} & \mathrm{r}=11 \text { to } 15 \\
\mathrm{~N}_{2}+\mathrm{O} \leftrightarrow \mathrm{NO}+\mathrm{N} & \mathrm{r}=16 \\
\mathrm{NO}+\mathrm{O} \leftrightarrow \mathrm{O}_{2}+\mathrm{N} & \mathrm{r}=17
\end{array}
$$

$M$ can be one of the five chemical species: $\mathrm{O}_{2}, \mathrm{~N}_{2}, \mathrm{NO}, \mathrm{O}$ or $N$ (in the order).

\section{AXISYMMETRIC FORMULATION}

We do not lose general information by seeking the solution at the points of an infinitely small domain Fig.1. A method developed within the Sinus project of the INRIA SophiaAntipolis [4] makes it possible to pass from $3 \mathrm{D}$ to $2 \mathrm{D}$ axisymmetric by using a technique of disturbance of domain. Taking advantage of this finding, here the problem is considered as being axisymmetric.

The system of equations (1) can be written as:

$$
\begin{aligned}
& \operatorname{mes}\left(C_{i, j}\right) \frac{\partial W_{i, j}}{\partial t}+\sum_{a \in\left\{x, x^{\prime}, y, y^{\prime}\right\}}\left(F_{i, j} \vec{i}+G_{i, j} \vec{j}\right) \cdot \vec{\eta}_{a}- \\
& H \text {.aire }\left(C_{i, j}\right)=\operatorname{mes}\left(C_{i, j}\right) \cdot \Omega_{i, j}
\end{aligned}
$$

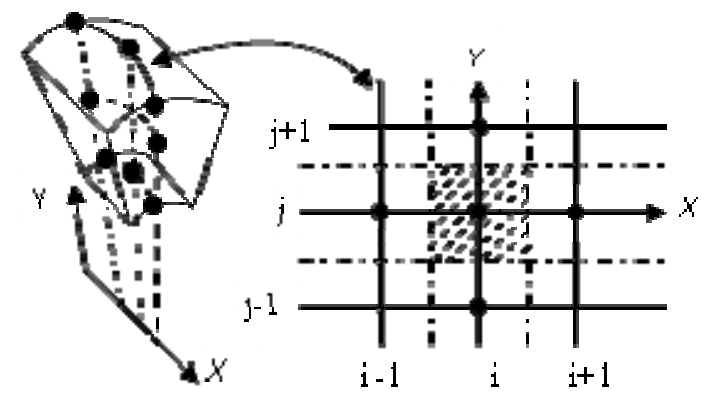

Fig. 1 Mesh form 
Where mes $\left(C_{i, j}\right)$ is the measurement (in $\left.\mathrm{m}^{3}\right)$ of an infinitely small volume of center $(i, j)$. aire $\left(C_{i, j}\right)$ is the surface of the symmetry plane passing by the center of elementary volume. $\eta_{a}$ is the integrated normal. For a detailed calculation of $\eta_{a}$, aire $\left(C_{i, j}\right)$ and $\operatorname{mes}\left(C_{i, j}\right)$ we refer to work of Goudjo [4]. The third term of the equation expresses the axisymmetric flow condition. Flows, $W, F, G$ and $H$ this time are given by:

$$
W=\left\{\begin{array}{l}
\rho \\
\rho u \\
\rho v \\
\rho e \\
\rho_{s} \\
\left(\rho_{v s} e_{v s}\right)_{O 2, N 2}
\end{array}\right\} \quad F=\left\{\begin{array}{l}
\rho u \\
\rho u^{2}+p \\
\rho u v \\
(\rho e+p) u \\
\rho_{s} u \\
\left(\rho_{s} e_{v s} u\right)_{O 2, N 2}
\end{array}\right\}
$$

$$
G=\left\{\begin{array}{l}
\rho v \\
\rho u v \\
\rho v^{2}+p \\
(\rho e+p) v \\
\rho_{s} v \\
\left(\rho_{s} e_{v s} v\right)_{O 2, N 2}
\end{array}\right\} \quad H=\left\{\begin{array}{l}
0 \\
0 \\
2 p \\
0 \\
0 \\
0
\end{array}\right\}
$$

$$
\Omega=\left\{\begin{array}{l}
0 \\
0 \\
0 \\
0 \\
\omega_{c S} \\
\left(\omega_{v S}\right)_{O 2, N 2}
\end{array}\right\}
$$

\section{DISCRETIZATION IN TIME}

The present numerical method is based on an explicit approach in time and space. The step of time $\Delta t$ is such as:

$$
\Delta t=\min \left(\frac{\Delta x \cdot C F L}{(\|V\|+a)}\right)_{i, j}
$$

The CFL (Courant, Friedrich, Lewis) is a stability factor. $V$ is the velocity of the flow and the speed of sound. $\Delta x$ is the small length of the mesh at the same point $(i, j)$. At each time step and for each point $(i, j)$, the system of equations (27) can be written as:

$$
\begin{aligned}
& W_{i, j}^{n+1}=W_{i, j}^{n}-\frac{\Delta t}{\operatorname{mes}\left(C_{i, j}\right)} \sum_{a \in\left\{x, x^{\prime}, y, y^{\prime}\right\}}\left(F_{i, j}^{n} \vec{i}+G_{i, j}^{n} \vec{j}\right) \cdot \vec{\eta}_{a}+ \\
& \Delta t \frac{\operatorname{aire}\left(C_{i, j}\right)}{\operatorname{mes}\left(C_{i, j}\right)} H_{i, j}^{n}+\Delta t . \Omega_{i, j}^{n}
\end{aligned}
$$

The choice of the grid plays an important role in determining in the convergence of calculations. Therefore, it is indeed advisable to have sufficiently refine meshes at the places where the gradients of the flow parameters are significantly large, as observed in front of the nose of the obstacle.

\section{DECOMPOSITION OF VAN-LEER}

In this study, the decomposition of Van-Leer [13] and [14] is selected, namely a decomposition of flows in two parts $F_{V L}^{-}$and $F_{V L}^{+}$.This decomposition must apply to the present two-dimensional problem by calculating the flow within each interface between two cells. Moreover, through this interface, the normal direction is paramount, thus, a change of reference mark is applied to place in the reference mark of the interface and its normal by the intermediary of a rotation $R$, Fig. 2 .

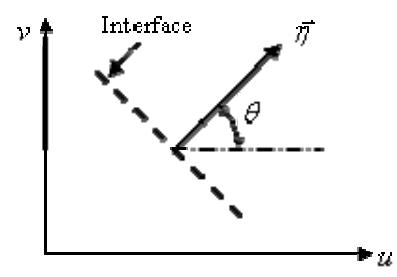

Fig. 2 Interface and its normal

The vector $W_{E} \quad$ (variable of Euler) is written $W_{E}^{R}$ in the new reference mark

$$
W_{E}^{R}=\left(\begin{array}{l}
\rho \\
\rho \overrightarrow{V_{n}} \\
\rho e
\end{array}\right)
$$

where $\overrightarrow{V_{n}}$ is obtained from $\vec{V}$, via the rotation $R$, in the following way:

$$
\vec{V}=\left(\begin{array}{l}
u \\
v
\end{array}\right) \rightarrow \overrightarrow{V_{n}}=\left(\begin{array}{l}
u_{n} \\
v_{n}
\end{array}\right)=\left(\begin{array}{cc}
\cos \theta & \sin \theta \\
-\sin \theta & \cos \theta
\end{array}\right)\left(\begin{array}{l}
u \\
v
\end{array}\right)
$$

where:

$$
\cos \theta=\frac{\eta_{x}}{\|\eta\|} \quad, \quad \sin \theta=\frac{\eta_{y}}{\|\eta\|}
$$




$$
\|\eta\|=\sqrt{\eta_{x}^{2}+\eta_{y}^{2}}
$$

The overall transformation $R$ is written overall

$$
\begin{aligned}
R & =\left(\begin{array}{cc}
\cos \theta & \sin \theta \\
-\sin \theta & \cos \theta
\end{array}\right) \\
R^{-1} & =\left(\begin{array}{cc}
\cos \theta & -\sin \theta \\
\sin \theta & \cos \theta
\end{array}\right)
\end{aligned}
$$

Moreover, at each interface $(i+1 / 2)$, two neighbor states (i) and $(i+1)$ are known. Thus, one can calculate the onedimensional flow $F$ through the interface, total flow $f(W, \vec{\eta})$ being deduced from $F$ by applying the opposite rotation, as:

$$
f(W, \vec{\eta})=\|\eta\| \cdot R^{-1}\left(F\left(W^{R}\right)\right)
$$

This property makes it possible to use only one component of flow $f$ ( $F$ for example) to define the decomposition of flow in two dimensions. Moreover, this method is much easy and simple to implement than the decomposition of flow in two dimensions $f=F \cdot \eta_{x}+G \eta_{y}$.

The expressions of $F_{V L}^{+}$and $F_{V L}^{-} \quad$ in 1-D which are those of $F_{V L}^{+}\left(W^{R}\right)$ and $F_{V L}^{-}\left(W^{R}\right)$, where $W^{R}$ is defined like the transform of $W$ by rotation $R$, can be written in the following from:

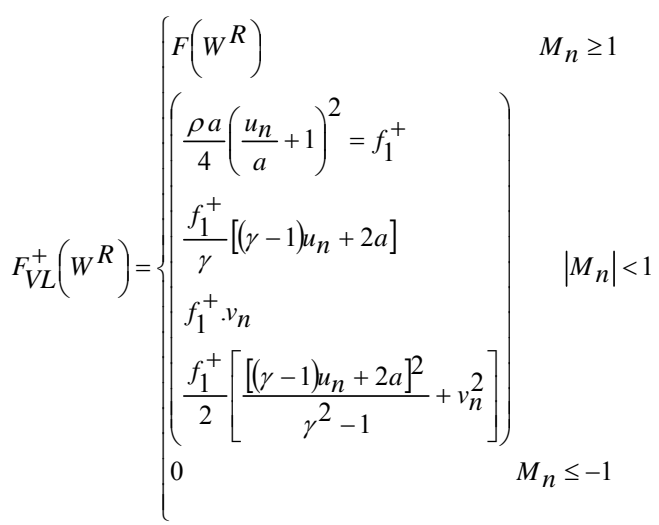

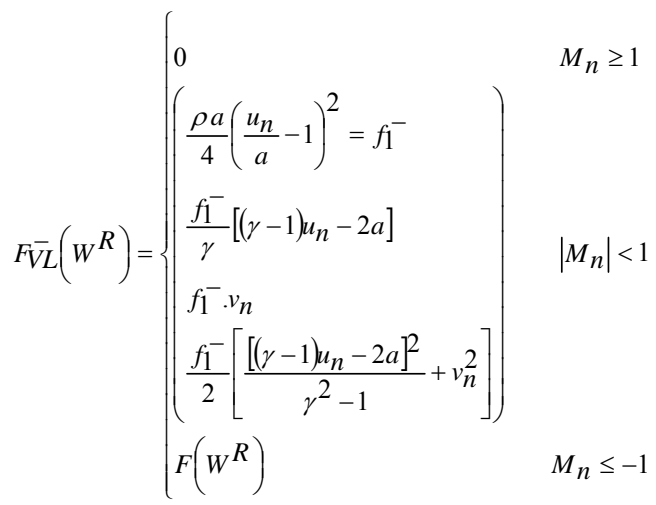

Where $M_{n}=\frac{u_{n}}{a}, \quad u_{n}$ and $v_{n}$ are the velocity in the reference mark of the interface.

\section{BOUNDARY CONDITIONS}

6.1 Border upstream

On the line of entrance, the parameters $M, p, T$ and $Y_{s}$ are fixed. Furthermore, as the flow reaches the entrance in a supersonic state, these parameters are unchanged during the iterations.

\subsection{Body surface}

In this case, as flow is not viscous, a slip condition is applied on the wall. At any point $\mathrm{M}(\mathrm{x}, \mathrm{y})$ on the wall the following condition must be checked:

$$
\vec{V} \cdot \vec{n}=0
$$

$\vec{n}$ is the normal to the wall.

\subsection{Axis of symmetry}

In any point $M$ of the axis of symmetry, the following condition should be satisfied:

$$
v=0 \rightarrow \vec{V}=u \vec{i}
$$

\subsection{Border downstream}

At the exit of the computational domain, downstream the body, the flow is supersonic; the exit values of the flow filed parameters are extrapolated from the interior values.

\subsection{Stagnation point}

At stagnation point we are two conditions, axis of symmetry and body surface. In this case we use the mesh presented in fig. 3. For calculation we impose:

$$
\begin{aligned}
& u(i, j-1)=u(i, j+1) \\
& v(i, j-1)=-v(i, j+1) \\
& u(i-1, j-1)=u(i-1, j+1) \\
& v(i-1, j-1)=-v(i-1, j+1)
\end{aligned}
$$


Because the coordinates points $(i, j-1)$ and $(i-1, j-1)$ do not exist. Meanwhile, we do not have the right to use symmetry with the wall. It is preferable to use a half-mesh and calculation must be carried out in the center.

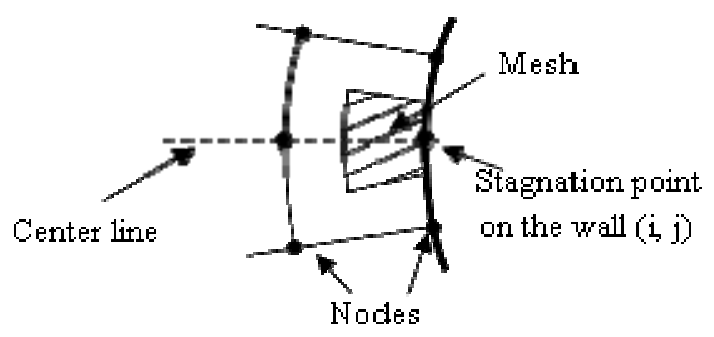

Fig. 3 Stagnation point mesh

\section{RESULTS AND INTERPRETATIONS}

The body employed for the numerical simulations is a hemisphere followed by a cylinder of $5^{\circ}$ of conical angle, as depicted in Fig.4. The grid used in calculations is composed of (40x180) nodes, for illustration, an example of grid of (20x90) is presented in Fig. 5. The composition of the air of the upstream flow is taken equal to $21 \%$ of $\mathrm{O}_{2}$ and $79 \%$ of $\mathrm{N}_{2}$. The pressure, and the temperature related to the air are function of the altitude. The Mach number is also given, see table III. For a flow detailed around the obstacle, one takes the case of an altitude of $45 \mathrm{Km}$. The pressure and the temperature related to the air at this altitude are $170 \mathrm{~Pa}$ and $295 \mathrm{~K}$, respectively. The Mach number is set to 10 .

Computations based on the $(20 \times 90)$ grid starts with the aerodynamic part which converged after 1000 iterations with a residue of $10^{-3}$. This is followed by complete scheme simulations where the residue reaches $10^{-6}$ and number of iterations equals 2600 iterations. Note that under the $(40 \times 180)$ grid, the iteration count increases up to 5400. The CFL is always taken equal to 0.4 .

For a flow without thermo chemistry consideration, the mass fractions of the chemical species and the energy of vibration are supposedly fixed. The residue of the relative variation of the density is about $10^{-5}$. There is no change in the findings for lower tolerance, say $10^{-8}$. Fig. 6 shows the variation of the residue according to the iterations count for a grid of $(40 \times 180)$ nodes. Fig. 7 illustrates the variation of the temperature along the axis of symmetry until stagnation point and then along the wall of the body. Difference between cases of a perfect gas (PG), and a non-equilibrium flow without coupling (V-T) and with coupling according to the model of Park (CVD) are presented.

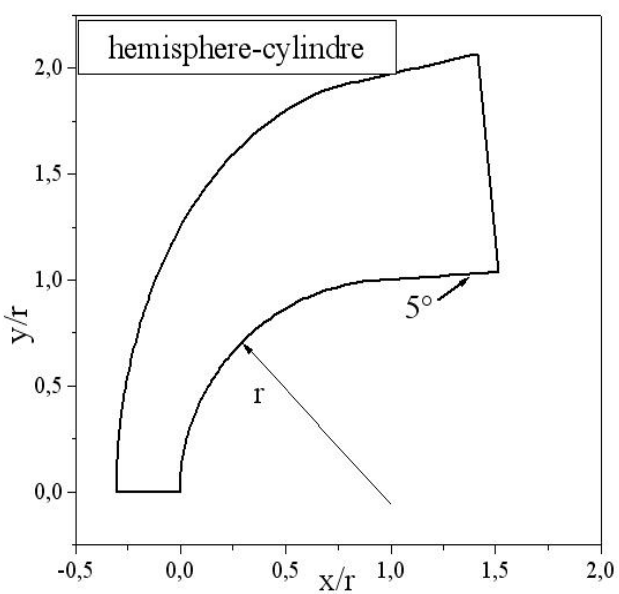

Fig. 4 Computational domain

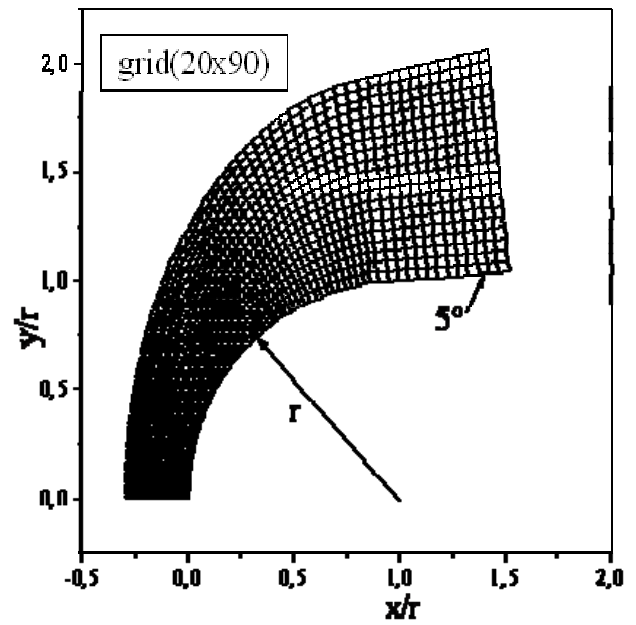

Fig. 5 Grid of (20x90)

Temperatures obtained in the case of the reactive flow are lower than those of perfect gas. At the stagnation point for example, the ratio of the temperature of the flow at the temperature of free stream is 17 instead of 21.3 . There is reduction of about $20.6 \%$ in comparison to a perfect gas. If we take account of coupling CVD by replacing the temperature $T$ by $T^{1-q} T_{v}^{q}$ with $q=0.3$, the difference is weak between this case and without coupling alternative.

Fig. 8 depicts the variation of the temperature of vibration of $\mathrm{O}_{2}$ and $\mathrm{N}_{2}$ compared with the temperature of translation rotation of the mixture. After the shock, the temperatures of vibration are strongly excited and after a distance of $\frac{x}{r}=1$ 
becomes frozen, $T_{V O 2}=12.7 T_{\text {inf }}, T_{V N 2}=8.55 T_{\text {inf }}$ and $T=7.53 T_{\text {inf }}$.

Fig. 9 and Fig. 10 illustrate the evolution of the mass fractions of the chemical species constituting the mixture of air. It is noticed that the dissociation of $\mathrm{O}_{2}$ and $\mathrm{N}_{2}$ starts just after the shock, their concentrations decrease up to the stagnation point and then a recombination will take place slowly, because the velocity of the flow is larger compared to the chemical kinetics.

A frozen of concentrations is obtained downstream where $\mathrm{Y}_{\mathrm{O} 2}$ becomes equal 0.19 instead of 0.21 and $\mathrm{Y}_{\mathrm{N} 2}$ becomes 0.78 instead of 0.79 . For $\mathrm{NO}, \mathrm{O}$ and $\mathrm{N}$ a fast formation of these species will occur after the shock up to the stagnation point and then a slow disappearance begins until having an inevitable freezing, where $Y_{N O}=1.16 \%, Y_{O}=0.9 \%$ and $Y_{N}=3.10^{-4} \%$. For more details see haoui [15].

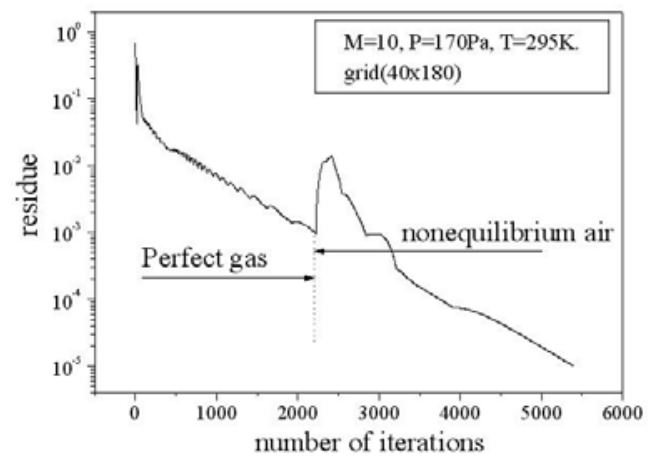

Fig. 6 Variation of residue

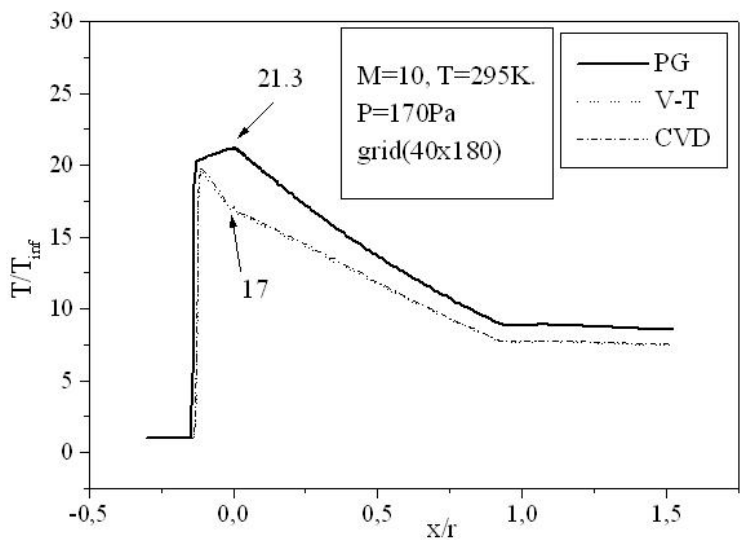

Fig. 7 Variation of temperature

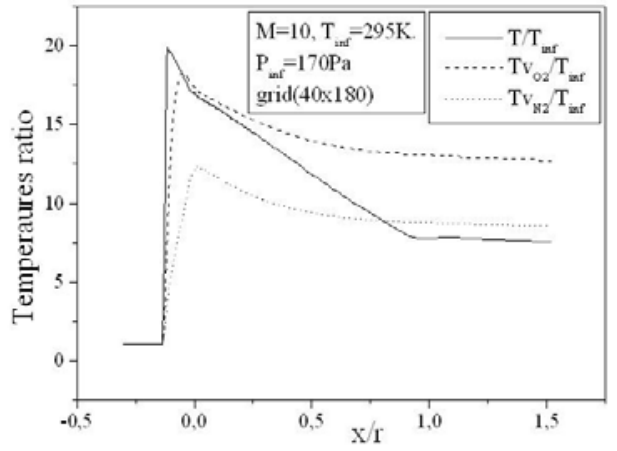

Fig. 8 Variation of temperature of vibration

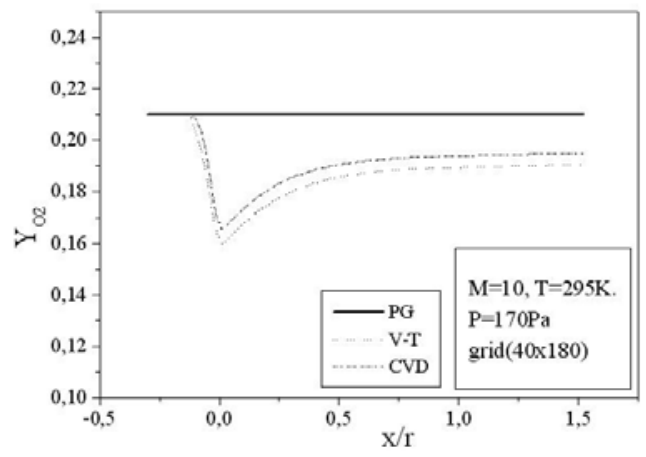

Fig. 9 Evolution of the mass fraction of $\mathrm{O}_{2}$

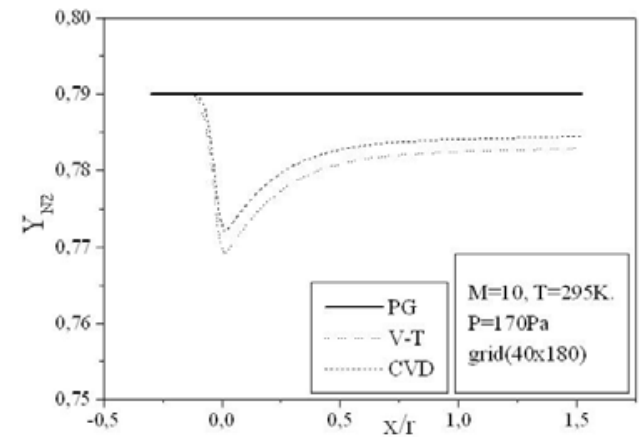

Fig. 10 Evolution of the mass fraction of $\mathrm{N}_{2}$ 
Concerning the flow far from the wall, we presented the Mach-contours around the body in fig. 11 where the detached shock wave is visible in front of the nose body. The position of the detached shock does not vary too much compared to the case without chemistry. For the non-equilibrium case, the position of the shock is at 0.135 times the radius in contrast to the case without chemistry ( 0.15 times the radius). Fig. 12 shows the temperature-contours around the body. At the stagnation point the temperature is $5015 \mathrm{~K}$, just after the shock it raises to $5870 \mathrm{~K}$; the difference is absorbed by the dissociation of the molecules. For the vibrational temperaturecontours of vibration of $\mathrm{O}_{2}$ and $\mathrm{N}_{2}$ and mass fraction-contours see haoui [15].

For the other phases of the trajectory one gives the results at the stagnation point according to altitude. The atmospheric pressure and temperature are known according to the altitude Fig. 13 and 14. Concerning the speed and the Mach number of free stream, we choose the data of a space shuttle Fig.15. After the execution of our program for various altitudes one obtained several information. The Fig. 16 shows the variation of the stagnation temperature according to altitude for two cases, perfect and real gas. One observes the great difference between the temperatures especially at high altitudes where the Mach number is great. For example, at $65 \mathrm{~km}$, the real temperature is $9000 \mathrm{~K}$ instead of $16000 \mathrm{~K}$ calculated with perfect gas. At $50 \mathrm{~km}$, the difference is due only to the vibration of the molecules. The Fig. 17 gives the composition of the air at the stagnation point according to altitude. The air is dissociated much more at high altitudes than in low altitudes. The Fig. 18 shows the difference between the translation rotation temperature and the vibrational temperatures, the flow is in nonequilibrium state even at the stagnation point.

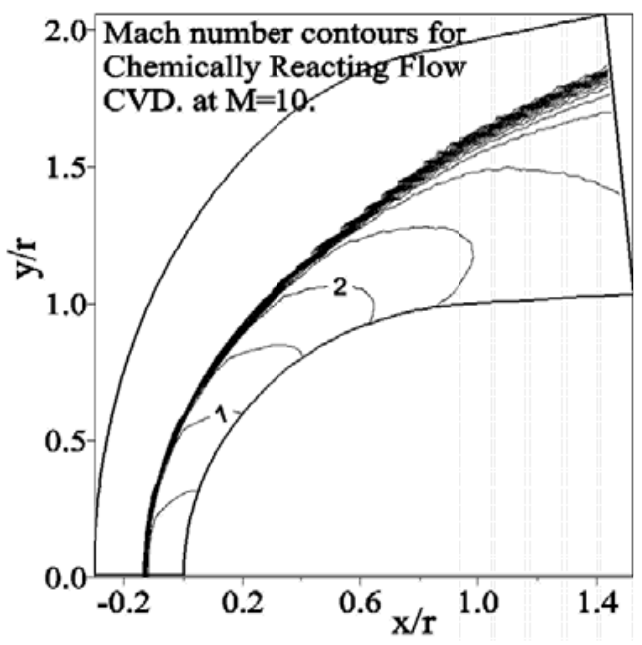

Fig. 11 Mach number contours

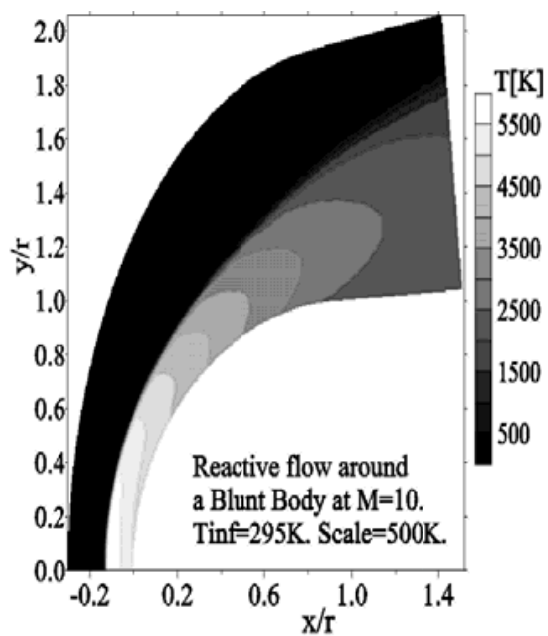

Fig. 12 Temperature contours

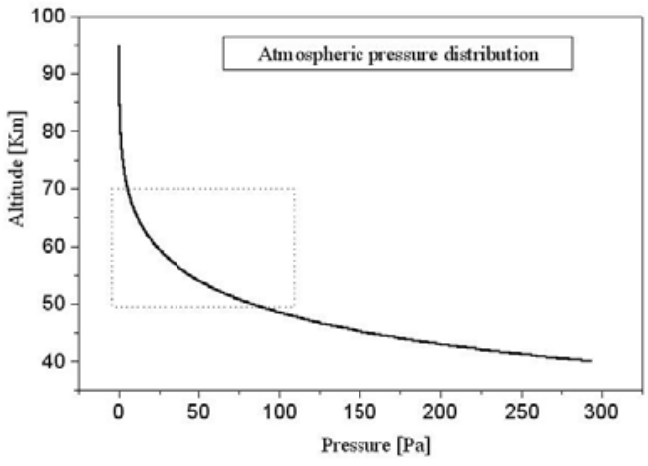

Fig. 13 Atmospheric pressure distribution 


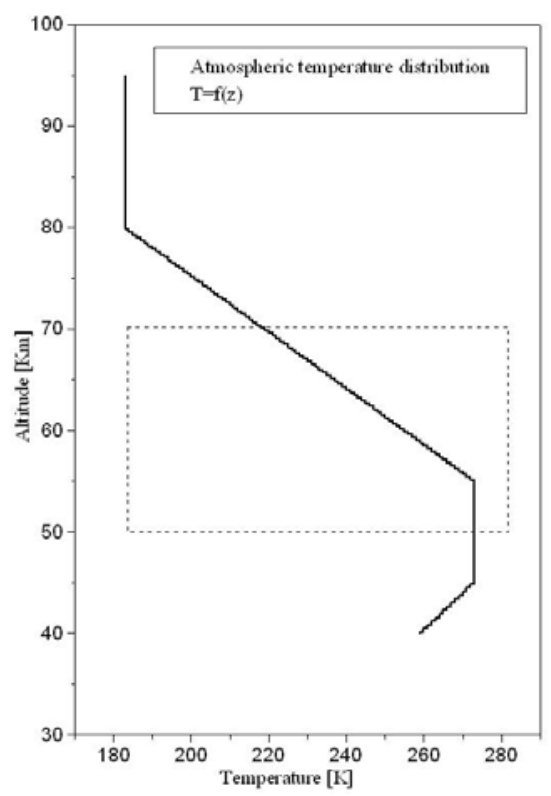

Fig. 14 Atmospheric Temperature distribution

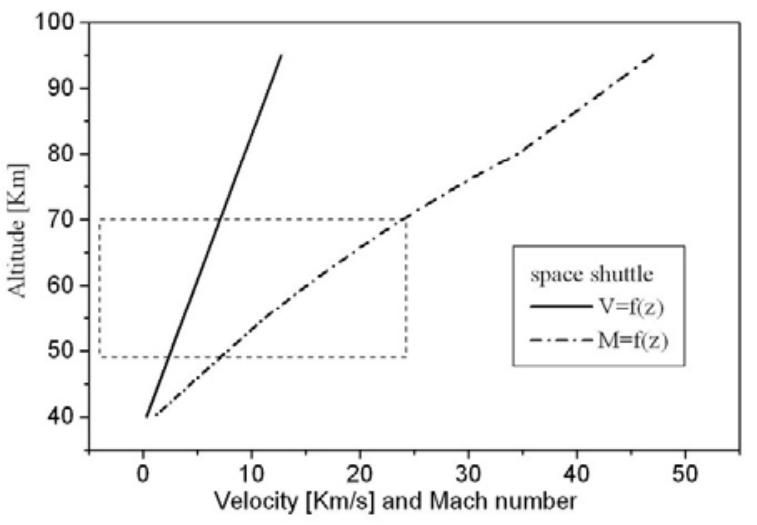

Fig. 15 Velocity and Mach number trajectory

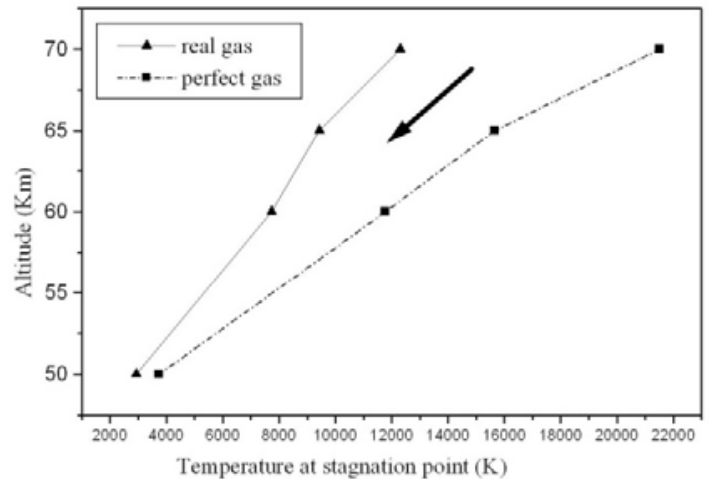

Fig. 16 Stagnation temperature along the trajectory

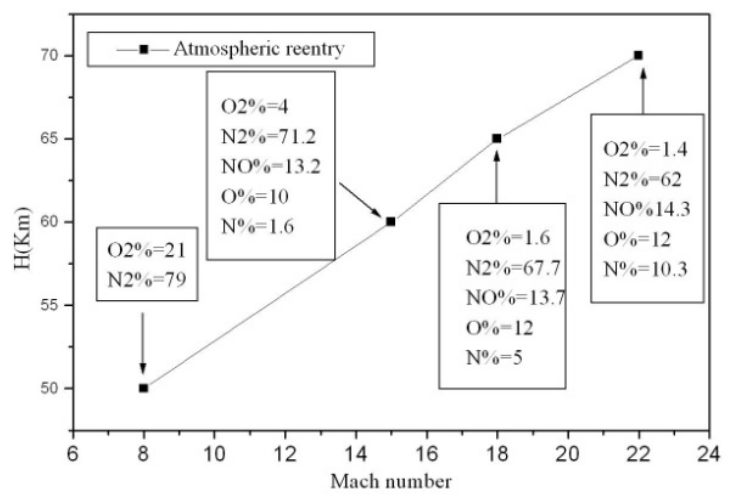

Fig. 17 composition mixture along the trajectory at the stagnation point

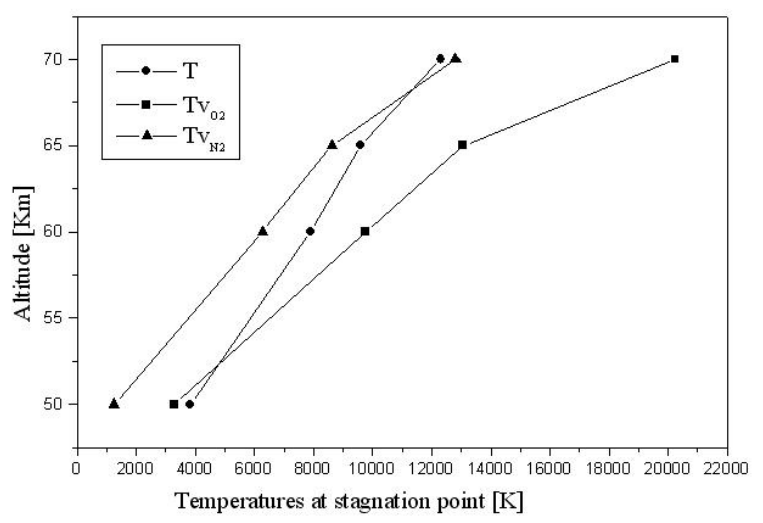

Fig. 18 Temperatures along the trajectory at the stagnation point

\section{CONCLUSION}

The numerical simulation of the flows around reentry bodies at high temperatures provided satisfactory results from a numerical and a physical point of view. With high degree of 
accuracy requirements, computational convergence is achieved and the physical phenomena considered are visible after the detached shock wave and around the blunt body. The choice of the kinetic model for this type of flows is interesting. The model with 17 reactions proves to be more realistic since it practically considers all the possible collisions between molecules and atoms of the air mixture. After the shock, the air is in nonequilibrium state, and a freezing is obtained after the stagnation point, about $\frac{x}{r}=0.6$. At the stagnation point of the body, the temperature of gas is found to be lower than a case where molecules dissociations are not implemented in the simulation. During the atmospheric reentry of spacecraft, when the altitude passes from $70 \mathrm{Km}$ to $50 \mathrm{Km}$, the temperature at the stagnation point passes from $12000 \mathrm{~K}$ to $3000 \mathrm{~K}$. The region surrounding the stagnation point is exposed to the high heat transfer. For example, at $45 \mathrm{~km}$ of altitude, the temperature at the stagnation point is $5100 \mathrm{~K}$, then it decreases along the wall up to the value $2000 \mathrm{~K}$ which corresponds to the distance $\mathrm{x} / \mathrm{r}=1$ from the stagnation point.

\section{REFERENCES}

[1] B. Van Leer, "Flux Vector Splitting for the Euler Equations," Lecture Notes in Physics. 170, (1982), 507-512.

[2] R. Haoui, A. Gahmousse, D. Zeitoun, "Condition of convergence applied to an axisymmetric reactive flow (Condition de convergence appliquée à un écoulement réactif axisymétrique)," 16th CFM, nº738, Nice, France, 2003

[3] R. Haoui, A. Gahmousse, D. Zeitoun, "Chemical and vibrational nonequilibrium flow in a hypersonic axisymmetric nozzle(Ecoulement hors d'équilibre chimique et vibrationnel dans une tuyère hypersonique axisymétrique), “ International Journal of Thermal Sciences, article $n^{\circ} 8$ , volume 40, (2001), pp787-795.

[4] Goudjo, J.A. Désidéri, "a finite volume scheme to resolution an axisymmetric Euler equations (Un schéma de volumes finis décentré pour la résolution des équations d'Euler en axisymétrique), “ Research report INRIA 1005,1989

[5] L. Landau, E. Teller, "Theory of sound dispersion," Physikalische Zeitschrift der Sowjetunion. 10, (1936), 34-43.

[6] Blackman, "Vibrational relaxation in Oxygen and Nitrogen," Technical Report, Palmer phys. Laboratory, Princeton University, 1955.

[7] K.L.Wray, "Shock tube study of the vibrational relaxation of Nitric Oxide," Journal of Chemical Physics. 36 (10) (1962) 2597-2603.

[8] W.C. Gardiner, "Combustion chemistry," Springer Verlag, NewYork, 1984

[9] S.H. Lee, "Electron impact vibrational excitation in the flow field of aero assisted orbital transfer vehicles," Progress in Astronautics and Aeronautics, Thermophysical aspects of re-entry flows. 103 (1986) $197-$ 224.

[10] J.H. Kiefer, R.W. Lutz, "The effect of Oxygen atoms on the vibrational relaxation of Oxygen," $11^{\text {th }}$ Symposium on combustion. Pittsburg, 1967 pp 67-74.

[11] W.D. Breshears, P.F. Bird, "Effects of Oxygen atoms on the vibrational relaxation of Nitrogen," The journal of chemical physics, 48 (10) (1968)

[12] F. Thivet, "Modeling of nonequilibrium of vibration in reactive flow (Modélisation du déséquilibre vibrationnel dans un écoulement réactif)," Management report, laboratory of molecular and macroscopic 10nergetic, combustion, CNRS/ECP, September-December 1989.

[13] Y. Burtschell, "Performances, dimensioning and numerical simulation of a hypersonic shock tunnel with reflected shock of free piston (Performances, dimensionnement et simulation numérique d'une soufflerie hypersonique à choc réfléchi à piston libre)," thesis of Doctorate, university of Aix-Marseilles I, France, 1990.

[14] M-C. Druguet, "Contribution to the study of nonequilibrium reactive hypersonic Euler's flows (Contribution à l'étude des écoulements eulériens hypersoniques en déséquilibre thermochimique)," Thesis of Doctorate. University of Provence, France, 1992.
[15] R. Haoui, "Finite volumes analysis of a supersonic non-equilibrium flow around the axisymmetric blunt body," International Journal of Aeronautical and space Sciences, 11(2), (2010), pp59-68.

TABLE I

FORWARD CONSTANTS. GARDINER MODEL

\begin{tabular}{|l|c|c|c|}
\hline Reactions & $\mathrm{A}$ & $\mathrm{n}$ & $\mathrm{T}_{\mathrm{d}}$ \\
\hline $\mathrm{r}=1$ & $1.64 \mathrm{e}+19$ & -1 & 59380 \\
$\mathrm{r}=2$ & $3.64 \mathrm{e}+18$ & -1 & 59380 \\
$\mathrm{r}=3$ & $1.82 \mathrm{e}+18$ & -1 & 59380 \\
$\mathrm{r}=4$ & $4.56 \mathrm{e}+19$ & -1 & 59380 \\
$\mathrm{r}=5$ & $1.82 \mathrm{e}+18$ & -1.6 & 59380 \\
$\mathrm{r}=6$ & $1.40 \mathrm{e}+21$ & -1.6 & 113200 \\
$\mathrm{r}=7$ & $3.70 \mathrm{e}+21$ & -1.6 & 113200 \\
$\mathrm{r}=8$ & $1.40 \mathrm{e}+21$ & -1.6 & 113200 \\
$\mathrm{r}=9$ & $1.40 \mathrm{e}+21$ & -1.6 & 113200 \\
$\mathrm{r}=10$ & $1.60 \mathrm{e}+22$ & -1.6 & 113200 \\
$\mathrm{r}=11$ & $4.00 \mathrm{e}+20$ & -1.5 & 75510 \\
$\mathrm{r}=12$ & $4.00 \mathrm{e}+20$ & -1.5 & 75510 \\
$\mathrm{r}=13$ & $8.00 \mathrm{e}+20$ & -1.5 & 75510 \\
$\mathrm{r}=14$ & $8.00 \mathrm{e}+20$ & -1.5 & 75510 \\
$\mathrm{r}=15$ & $8.00 \mathrm{e}+20$ & -1.5 & 75510 \\
\hline $\mathrm{r}=16$ & $1.82 \mathrm{e}+14$ & 0 & 38370 \\
$\mathrm{r}=17$ & $3.80 \mathrm{e}+9$ & 1. & 20820 \\
\hline
\end{tabular}

TABLE II

EQUILIBRIUM CONSTANTS

\begin{tabular}{lcrrrr}
\hline reactions & $\mathrm{c}_{0}$ & \multicolumn{1}{c}{$\mathrm{c}_{1}$} & $\mathrm{c}_{2}$ & \multicolumn{1}{c}{$\mathrm{c}_{3}$} & \multicolumn{1}{c}{$\mathrm{c}_{4}$} \\
\hline $\mathrm{r}=1$ à 5 & 1.335 & -4.127 & -0.616 & 0.093 & -0.005 \\
$\mathrm{r}=6$ à 10 & 3.898 & -12.611 & 0.683 & -0.118 & 0.006 \\
$\mathrm{r}=11$ à 15 & 1.549 & -7.784 & 0.228 & -0.043 & 0.002 \\
\hline $\mathrm{r}=16$ & 2.349 & -4.828 & 0.455 & -0.075 & 0.004 \\
$\mathrm{r}=17$ & 0.215 & -3.658 & 0.843 & -0.136 & 0.007 \\
\hline
\end{tabular}

TABLE III

CHARACTERISTICS OF FREE STREAM ALONG THE TRAJECTORY

\begin{tabular}{|l|r|r|r|r|}
\hline $\mathrm{H}(\mathrm{Km})$ & 70 & 65 & 60 & 50 \\
\hline $\mathrm{P}(\mathrm{Pa})$ & 5.52 & 14.01 & 22.5 & 79.8 \\
\hline $\mathrm{T}(\mathrm{K})$ & 220 & 238 & 256 & 271 \\
\hline Mach & 22 & 18 & 15 & 8 \\
\hline
\end{tabular}

